\title{
Scheduling of nonconforming devices: the case of a company in the automotive sector
}

\begin{abstract}
This article presents a project developed in a company's quality department aiming at scheduling nonconforming devices analysis process. The company faced a problem of no compliance with pre-established time requests with a huge impact in terms of fines paid to its demanding customers of the automotive sector.

In order to overcome this problem, scheduling tools were developed and tested. The problem found in the scheduling is characterized as: the objective is to minimize the number of tardy tasks in identical parallel machines.

The simulation of different scheduling rules allowed to confirm that the current prioritization rule is not the most effective one. Preliminary simulation were carried out using Lekin software showing that others criteria promote better results. According to the results achieved from the new developed algorithm that combines two criteria, it was obtained a $7 \%$ reduction in the analysis times and consequently a reduction on the fines paid to the clients.

Despite the preliminary status of present results, it is possible to foresee some improvements in the analysis process performance by using decision making support tools based on scheduling algorithms. This way, a significant improvement on the number of analysis which fulfill the defined pre-requirements will be achieved.
\end{abstract}

Keywords: Quality, Complaints, Priorization, Scheduling, Lekin.

\section{Introduction}

With market globalization and the increase of competition in the industrial sector, product quality has gradually become a key factor in a company's success (Colledani \& Tolio, 2006). High consumer demand and global competition has been forcing companies to continuously improve the quality level in its products and services (Pešić, Milić, \& Stanković, 2012), so they can provide effective and efficient support services to their clients according to their needs (Murali, Pugazhendhi, \& Muralidharan, 2016).

In order to improve the customers' support service, it is essential that companies possess a complete vision of all the customer's processes and are therefore able to identify and analyze all the relevant information to aid in their management and planning (Dombrowski \& Malorny, 2016). Since planning is a rather complex problem that directly influences a service's performance, it should be supported by an efficient scheduling of the tasks that constitute it. While planning concerns about the tasks to be done, scheduling concerns about the when and who carries the tasks out (Dean \& Kambhampati, 1997).

Scheduling is an important decision making process and widely used in the production systems of many companies. Scheduling consists in the allocation of 
resources throughout determined time periods and its goal is to optimize one or more objectives. Tasks in the scheduling process are characterized by:

1) a certain level of priority; 2) an earliest possibly starting date; 3 ) an effective date for beginning; 4) a processing time; 5) a due date and 6) a deadline. The goal of using a scheduling process is to minimize or maximize the objective function, so that the company is able to obtain good solutions and reach its goals (Pinedo, 2008).

The project presented in this article was developed in an industrial environment, in a company's Warranty Laboratory and it arose with the necessity of improving the complaints process for nonconforming devices, because the analysis times of nonconforming devices wasn't in compliance with the pre-established temporal requests. The noncompliance of the mentioned requests, particularly the established timeline for the identification of the origin of the malfunction, involves the payment of heavy financial fines for the company.

This project proposes the definition of sequencing rules and prioritization of the devices to be analyzed, with the objective of improving the complaints process and consequently minimize the number of analysis done in the established timeline, diminishing the fines paid to the clients.

The remainder part of the paper is organized as follows. Section 2 presents a literature review on Scheduling problems. Section 3 presents the main problem and its characterization according to scheduling problems. Section 4 illustrates the applicability of the scheduling model and discussion of the main results of the project. Finally, Section 5 reports the main conclusions, limitations and final considerations regarding future developments.

\section{Literature review}

A complaints service plays a key part in organizations in order to obtain and recover customer satisfaction (Lovelock, Wirtz, Keh, \& Lu, 2002), so offering adequate responses to the complaints services is necessary in order to correct problems and keep the customers as service providers (Quy, 2014). Supplying an adequate customer support service is the key "ingredient" in product quality and competitive success (Cohen \& Lee, 1990). Due to the current competitive environment among companies, scheduling has been becoming a need for their survival, once companies have to increasingly comply with the customers' established response times (Pinedo, 2008).

Scheduling is a process optimization in which limited resources are allocated through time between parallel and sequential activities. Such situations occur in factories, editors, transportations, universities, hospitals, airports, warranty services, among others (Bagchi, 1999). Scheduling deals with the allocation of scarce resources and activities with the objective of optimizing one or more performance indicators (Leung, 2004).

Due to the varied possible combinations between different machine environments, different objectives and different side constraints, hundreds of 
useful scheduling problems can be obtained. As a result of this abundance of problems, as well as the constant attention of investigators from different optimization areas and operational research, throughout the last decades, scheduling literature has become rather extensive (Dean B. C., 2005). Scheduling currently represents a knowledge basis about models, techniques and knowledge on real systems. Considering scheduling as if it includes pure allocation problems, the formal development of techniques and optimization models for modern scheduling theories probably started in the years previous to World War II. In 1950 the first formal articles began to be recognized about characteristics of scheduling problems and only in 1960 did the first books arise (Baker \& Trietsch, 2009). During the 1970s and 1980s a greater abundance of articles and books about scheduling appeared, examples being Coffman (1976) and French (1982) that focus on deterministic scheduling aspects. Since then different and diverse scheduling problems articles have been developed (Baker \& Trietsch, 2009; Pinedo, 2008).

According to Pinedo (2008), a scheduling problem presents a taxonomy described according to three classes, $\alpha|\beta| \gamma: \alpha$ describes the machine environment and contains a single entry, $\beta$ describes details about characteristics and processing restrictions, containing a single or multiple entries and $\gamma$ describes the optimization criteria or performance measures.

In the last decades, investigators have given more attention and relevance to the study of priority rules (Blackstone, Philliphs, \& Hogg, 1982; Oliver \& Rajendran, 1997). Priority rules are used to select the following task to be processed from a set of tasks that await the availability of a machine.

Some examples of priority rules are Shortest Process Time (SPT), that assigns priority to the tasks that have the shortest processing time in that machine, Earliest Due Date (EDD) that assigns priority to the task with the earliest due date, Longest Processing Time (LPT) that assigns priority to the task with the longest processing time in that machine, First-In-First-Out (FIFO), where the task that arrived first will be processed first, Minimum Slack (MS) assigns the task that possesses the shortest slack time until the delivery date, Earliest Release Date (ERD) assigns the latest task to be entered in the system (Barksdale, Powell, \& Hargrove, 1984; Carvalho, 2000).

Scheduling problems in parallel machines environments have been studied throughout several years due to its importance in investigation and industrial environments, since the delay of tasks relating to their limit conclusion time is considered to be an essential indicator in measuring its performance (Armentano \& Yamashita, 2000). Even after recognizing and studying problems with the objective of minimizing tardiness, such as minimizing the sum of tardy tasks, there have been fewer progress in the problems regarding minimizing the number of tardy tasks in a parallel machine environment (Ho \& Chang, 1995; Shim \& Kim, 2007). In scheduling problems for parallel machines there usually are two decisions that need to be taken. One consists in allocating tasks to specific machines and the other regards determining the sequence of tasks in each machine (Shim \& Kim, 2007). 


\section{The Problem}

In the case study company, the complaint process of a nonconforming device is divided in 3 stages: customer complaint, where the client detects the device failure and reclaims the device to the company; the analysis process, where the device is analyzed in the Warranty Laboratory that looks for the root cause of the failure and finally the response to the client, communicating about the reported problem. This process has a customer response time for the complaint based on company standards and customer requests.

This project will focus on the stage of the analysis process of nonconforming devices in the Warranty Laboratory defined in Figure 1.

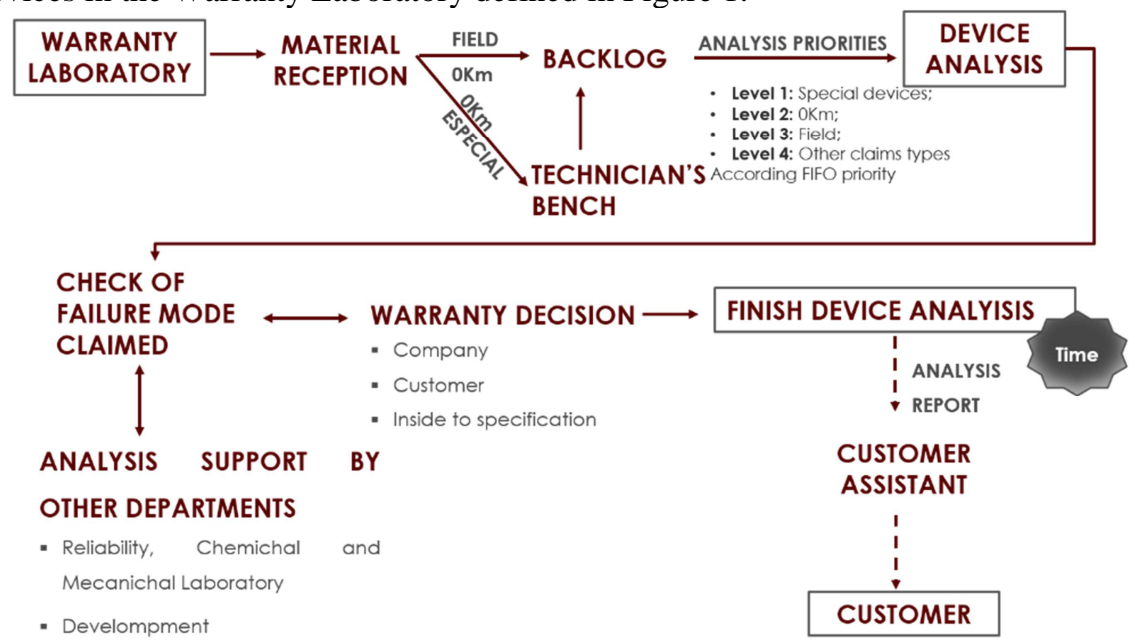

Figure 1 - Analysis process of nonconforming devices

There are many cases of nonconforming devices not fulfilling the company's and the clients' standards in the responses in its analysis in the Warranty Laboratory. This can be related to several factors such as: i) the priorities of analysis are not adequate to the temporal request of the clients and simply follow FIFO; ii) technicians that perform the analysis don't comply with the priority orders indicated by the system currently used in the company and iii) the high Backlog time (waiting time before analysis) for the nonconforming devices.

In 2015 only 1099 out of 2736 (40\%) of the clients complaints complied with the response times according to the company's standards. Therefore, the indemnity values paid to the customers in 2015 was of millions of euros.

In order to apply scheduling in the analysis process of nonconforming devices, a characterization was needed according to the characteristics of scheduling problems:

- Nature of variables: stochastic scheduling, since the analysis time can have uncertain / variable values;

- $\quad \alpha$ - Machine environment: identical parallel machine problem, $\alpha=$ Pm, since the technicians responsible for each group possess the same expertise and similar speed executing the tasks; 
- $\quad \beta$-Characteristics and processing restrictions:

- Release Date, $\beta=\mathrm{rj}$, the analysis of a nonconforming device can only be initiated the moment a device enters the system; it can be interrupted;

○ Interruptions, $\beta=$ prmp, a device's analysis is not continual since

- Machine eligibility restrictions, $\beta=\mathrm{Mj}$, when a result is sent to the Warranty Laboratory after a support in the analysis from another department, the resuming of the analysis will be attributed to the technician that started the analysis.

- $\quad \gamma$ - Performance measures or optimization criteria:

$\circ \quad$ Minimize the number of tardy jobs, $\gamma=\operatorname{Min}(\Sigma w j U j)$.

\section{Solutions and results}

Scheduling tools are necessary in order to propose improvements relative to the established priorities currently used in the Warranty Laboratory. Lekin software was developed by the Stern School of Business (1998), which contains a series of Scheduling algorithms and heuristics that allow the user to test its heuristics. Data regarding the Warranty Laboratory was tested with the goal of verifying which rule or priority criteria allowed to obtain the best result relative to the objective functions, which is the least weighted number of tardy tasks. It was however verified that Lekin software possesses some limitations, so it was necessary to simplify the characteristics of the analysis process of nonconforming device presented in Section 3.

According to the $\alpha|\beta| \gamma$ notation, the problem simulated on Lekin presents the following characteristics: $\alpha=$ Parallel Identical Machines, Pm; $\beta=$ Release Date, rj; $\gamma=$ minimize the weighted number of tardy tasks, Min $(\Sigma w j U j)$.

The following conditions were taken into account in the simulations on software Lekin:

- $\quad$ Consider a single work group of the Warrant Laboratory, technicians =4;

- Perform the simulation in a determined time period;

- Attribute 7 daily hours per technician;

- Consider the analysis time for each task according to the assignment made by the Laboratory coordinator and by the 4 technicians before the beginning of the analysis;

- Only select analysis that depend entirely on the Warranty Laboratory, since Lekin doesn't allow interruptions or restrictions in the choice of technician in allocating the task;

- Attribute different weighs to the tasks according to the importance that the company attributes to the type of customer complaint.

Three simulations were ran on Lekin with the objective of obtaining a better solution relative to the result obtained by the current priority criteria. The following priority criteria were tested: First-In-First-Out, FIFO, Earliest Due Date, EDD, Minimum Slack, MS, e Shortest Processing Time, SPT. Figure 2 shows the Gantt diagram referring to the first Lekin software simulation for each used criteria. 


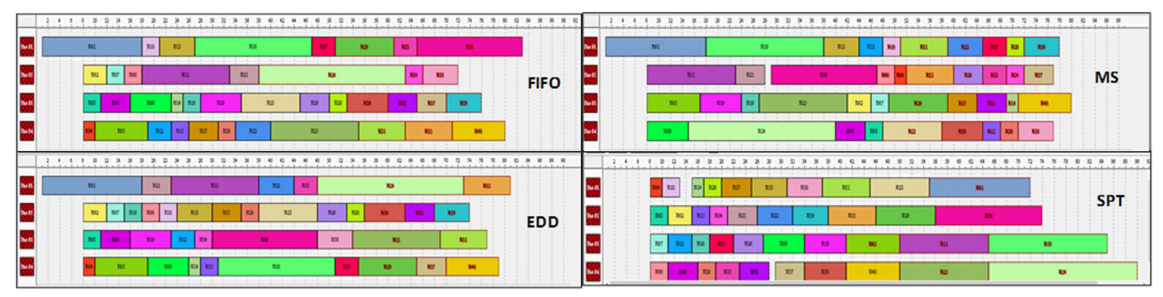

Figure 2 - Simulation 1 on Lekin software

It is concluded (Figure 3) that the priority criteria currently used in the Laboratory is not the one that obtains the best results for the selected objective function (number of tardy tasks). The following data supports this conclusion:

- In the first simulation, the FIFO criteria results in $32,5 \%$ tardy tasks, with EDD resulting in only $25 \%$ and SPT $17,5 \%$;

- The second simulation shows the percentage of tardy tasks goes from $58 \%$ to $37,5 \%$ if the EDD or SPT criteria is used instead of FIFO;

- $\quad$ The third simulation shows that FIFO causes $25 \%$ tardy tasks while EDD only causes $5 \%$;

- In the total of the 3 simulations, EDD has 23,8\% tardy tasks, SPT has $25 \%$, FIFO has $38 \%$ and MS $50 \%$.

We can the conclude that the rules that achieve the best results according to the simulation ran on Lekin are EDD and SPT. These results happen when no new tasks arrive until all the tasks in analysis are concluded.

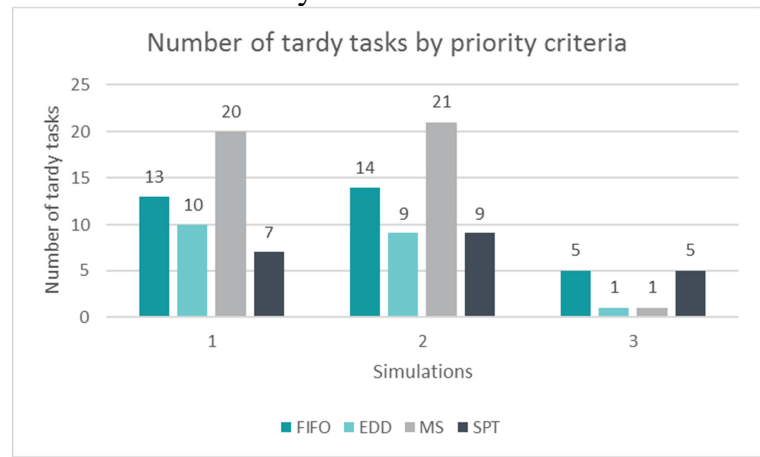

Figure 3 - Number of tardy tasks by priority criteria

This software possesses some limitations, so it was necessary to develop a tool that allowed adding more inherent characteristics to the process under analysis.

A scheduling algorithm has been proposed with the objective of finding a better solution for the prioritization in the analysis of nonconforming devices and consequently obtains better results in the amount of tardy tasks.

The definition of prioritization and process scheduling rules used in the algorithm are based in the Moore and Hodgson Algorithm (French, 1982) and in the results obtained in the simulations done in Lekin, where EDD and SPT criteria proved to obtain the best results.

The scheduling algorithm takes into account the combination of two priority criteria. EDD orders the device list in order to allocate the technicians and later 
assign priorities to the devices with larger weight. SPT influences the weight attributed to the devices. Other than the SPT criteria, the weight of devices is also influenced by the type of customer complaint and the state of the analysis.

The first stage was the characterization of the problem according to the scheduling classes $\alpha|\beta| \gamma: \alpha=$ Parallel Identical Machines, Pm; $\beta=$ Release Date, $\mathrm{rj} ; \beta=$ preemption, prmp e $\beta=$ machine eligibility restrictions, Mj; $\gamma=$ minimize the weighted number of tardy tasks, Min $(\Sigma w j U j)$.

The second stage was the description of each $\mathrm{j}$ task's data according the notation of scheduling problems.

The third stage was the definition of the moment the scheduling algorithm should be generated for analysis in the Warranty Laboratory.

The fourth stage was to mathematically define the algorithm rules.

Lastly, the algorithm was simulated using JAVA language.

Some considerations have to be taken into account in the algorithm's functioning:

- In case the machines have the same availability date, the algorithm randomly chooses one of the machines;

- In the first time, the algorithm allocates the seven first work hours of the technician and later allocates the tasks to the technicians with the earlier available date, meaning the technician is attributed a new task whenever he finishes one.

- If the real analysis time exceeds the estimated time, there is no weighting in the decision, meaning the technician must finish the device his or hers current analysis before starting a new analysis, or start analyzing a new device and reschedule the device in standby to the same technician. The current decision is to continue the analysis until it is finished.

This algorithm allows the Lekin software to:

- $\quad$ Schedule tasks submitted to analysis in other departments;

- $\quad$ Schedule according to EDD and SPT criteria;

- Schedule if the technician finishes the analysis earlier or later than the scheduled date readjusting his tasks to the moment he becomes free, meaning he or she can't start a new analysis if he or she hasn't finished the previous task;

- Schedule taking into account the estimated analysis times and reschedule in case the real analysis time differs the estimated time.

The programmed algorithm was tested and compared to the data obtained was compared to the data generated by the algorithm currently in place in the Warranty Laboratory (Table 1).

Table 1 - Comparison of results according to algorithm and Warranty Laboratory priorities

\begin{tabular}{|c|c|c|c|}
\hline & Delayed Tasks & Tasks done on time & Total tasks \\
\hline Algorithm Priority & 3 & 28 & 31 \\
\hline $\begin{array}{l}\text { Warranty } \\
\text { Laboratory Priority }\end{array}$ & 5 & 26 & 31 \\
\hline
\end{tabular}


Based on the data presented in Table 1, it is concluded that the number of delayed tasks according to the priorities of the algorithm is $3(9,67 \%)$, therefore lower than the number of tardy tasks according under the QMM-FA priorities, which was $5(16,12 \%)$.

\section{Discussion and conclusion}

The process of analysis of nonconforming devices is concluded to be very complex and to possess great variability in the analysis times as well as in the operations made during the analysis. Such process variability influences the results obtained in this project.

The simulations of several priorities rules in Lekin software have allowed to obtain different types of scheduling in the analysis of the technicians. Of the obtained results, it was concluded that EDD and SPT produced the best results. The rule currently used in the Warranty Laboratory, FIFO, obtained worst results than the previously defined criteria.

The EDD criteria resulted in $23,8 \%$ of delayed tasks, SPT in $25 \%$, FIFO in $38 \%$ and MS $50 \%$.

Only using the EDD criteria instead of FIFO results in a $14 \%$ reduction in the number of delayed tasks.

Comparing the obtained results with the algorithm developed in this project with the results obtained in Lekin software, it is concluded that the developed algorithm allows the scheduling of tasks with support analysis from other departments, with real and estimated times and also the fact that the EDD criteria was joined with the SPT; besides it makes it so that new tasks can be scheduling and allows the association of a new task in case the technician finishes earlier.

The implementation of this algorithm for the prioritization of analysis in the Warranty Laboratory can be said to bring significant improvements relative to the number of tardy tasks and and consequently a reduction on the fines paid to the clients, since there is a $7 \%$ improvement relatively to the current prioritization. However, it is necessary to take into account that the obtained results are preliminary and in need of further validation by widening the number of tests and performing a data sensibility analysis.

Throughout this project some limitation were felt that directly or indirectly affected the obtained results. One of the larger difficulties was the fact that the complaints process has some activities that are not completely defined. Besides, the variability and uncertainty in the analysis time, in the root cause associated with the complained defect, in the description of the complained defect, in the different tests realized during the analysis, in the type of support provided by other departments and in the data in the systems meant a limitation that stopped the project from obtaining better results. Another limitation was the fact that the company didn't provide access to the external costs of the clients' indemnities, making it impossible to measure the impact of this project in cost reduction.

Future work will start by increasing the number of algorithm tests, a sensitivity data analysis and improvement heuristics will be applied. 


\section{Acknowledgements}

This work has been supported by COMPETE: POCI-01-0145-FEDER-007043 and FCT - Fundação para a Ciência e Tecnologia within the Project Scope: $\mathrm{UID} / \mathrm{CEC} / 00319 / 2013$.

\section{References}

[1] Armentano, V., \& Yamashita, D. (2000). Tabu search for scheduling on identical parallel machines to minimize mean tardiness. Journal of Intelligent Manufacturing, 11, 453-460.

[2] Bagchi, T. (1999). Multiobjective Scheduling by Genetic Algorithms. Kluwer Aademic Pubishers.

[3] Baker, K., \& Trietsch, D. (2009). Principles of Sequencing and Scheduling. A John Wiley \& Sons, Inc. Publication .

[4] Barksdale, H., Powell, T., \& Hargrove, E. (1984). Complaint voicing by industrial buyers. Industrial Marketing Management, 13, 93-99.

[5] Blackstone, J., Philliphs, D., \& Hogg, G. (1982). A state-of-the-art survey of dispatching rules for manufacturing job shop operations. International Journal of Production Research 20, 27-45.

[6] Carvalho, D. (2000). Programação da Produção. Universidade do Minho.

[7] Coffman, E. (1976). Computer and Job-Scheduling Theory. Wiley.

[8] Cohen, M. A., \& Lee, H. L. (Winter 1990). Out of Touch with Customer Needs? Spare Parts and After Sales Service. Sloan Management Review, 31(2), 55.

[9] Colledani, M., \& Tolio, T. (2006). Impact of Quality Control on Production System Performance. CIRP Annals - Manufacturing Technology, 453-456.

[10] Dean, B. C. (2005). Approximation Algorithms for Stochastic Scheduling Problems. Massachusetts Institute of Techonology.

[11] Dean, T., \& Kambhampati, S. (1997). Planning and Scheduling.

[12] Dombrowski, U., \& Malorny, C. (2016). Process Identification for Customer Service in the field of the after Sales Service as a Basis for "Lean After Sales Service. Procedia CIRP 47, 246-251.

[13] French, S. (1982). Sequencing and Scheduling: And Introduction to the Mathematics of the JobShop. Ellis Horwood, LTD.

[14] Ho, J. C., \& Chang, Y.-L. (1995). Minimizing the number of tardy jobs. European Journal of Operation Research, 84(2), 343-355.

[15] Leung, J. (2004). Handbook of scheduling: Algorithms, Models and Performance Analysis. CHAMPMAN \& HALL/CRC

[16] Lovelock, C., Wirtz, J., Keh, H., \& Lu, X. (2002). Services Marketing in Asia, Managing People, Techonology ans Strategy. Singapore: Prentice Hall International Incorporation.

[17] Murali, S., Pugazhendhi, S., \& Muralidharan, C. (2016). Modelling and Investigation the relationship of after sales service quality with customer satisfaction, retention and loyalty - A casa study of home appliances business. Journal of Retailing and Consumer Service, 30, 67-83.

[18] Oliver, H., \& Rajendran, C. (1997). Efficient dispatching rules for scheduling in a job shop. Internationa Journal of Production economis, 48, 87-105.

[19] Pešić, M. A., Milić, V. J., \& Stanković, J. (2012). Significance of business quality management for increasing competitiveness of serbian economy. Serbian Journal of Management, 7(1), 149170.

[20] Pinedo, M. L. (2008). Scheduling: Theory, Algorithms, and Systems ( $3^{\circ}$ ed.). Prentice Hall.

[20] Quy, V. T. (2014). The impact of Organizational Responses to Complaints on Posto Purchase Behavioral intentions via Recovery Satisfaction - The Case of Saigon commercial Bank. Strategic Management Quartely, 2, 44-79.

[21] Shim, S.-O., \& Kim, Y.-D. (2007). Scheduling on parallel identical machines to minimize total tardiness. European Journal of operation research, 177(1), 135-146. 
[22] Stern School of Business, NYU. (1998). LEKIN -- Scheduling System. Obtido em 12 de 07 de 2016, de LEKIN® - Flexible Job-Shop Scheduling System: http://webstatic.stern.nyu.edu/om/software/lekin/

[23] Varela, L. (2007). Uma Contribuição para o Escalonamento da Produção baseado em Métodos Globalmente Distribuídos. Universidade do Minho. 